\title{
The rate of sample rejection and pre-analytical errors at KCMC Clinical Laboratory in Moshi, Kilimanjaro
}

\author{
Victor V Moshaa, Claudia Kabanyanab \\ -Department of Biotechnology Laboratory, Kilimanjaro Clinical Research Institute (KCRI), Moshi, Tanzania. \\ bKilimanjaro Christian Medical University College (KCMUC), Moshi, Tanzania. \\ *Correspondence to Victor V Mosha (victormosha@ymail.com)
}

\begin{abstract}
Background: Over the years, modern medicine has provided better quality services to patients. However, medical error is still prevalent and has a lot of negative consequences on patients' outcomes. These may include delayed treatment, longer hospital stays, or even worsening of the patient's condition. This study aimed to determine the sample rejection rate at KCMC Clinical Laboratory and characterize pre-analytical errors that contribute to it.

Methods: Data extraction sheet was used to collect information from rejection forms from January to December 2016. The data collection form gathered information on where samples were collected and send for laboratory analysis, and the types of rejected sample. Information on the reasons for sample rejection were also collected.
\end{abstract}

Results: The proportion of rejected samples stand at $0.19 \%$ lout of 117181 samples received from January to December 2016, 234 were rejected). The highest rates of rejection were from haematology section 78 (33.3\%). The major type of rejected sample was blood $(86.3 \%)$ and $20 \%$ of the rejected samples came from internal medicine department, mainly from its inpatient department (13.6\%). The commonest reason for rejection was unpaid specimens 84 (35.9\%).

Conclusion: Study results indicate the need for improving quality control system which will enhance sample management and prompt identification of deficiencies. The high rate of sample rejection due to lack of payment signify the need for improved government policies that address health expenses coverage. Provision of trainings on phlebotomy and blood samples transportation can reduce the rate of sample rejection due to clotting.

Keywords: Clinical laboratory, Pre-analytical errors, Rejected samples, Rejection rate.

\section{INTRODUCTION}

Clinical laboratory services play a significant role in the management of patients. Clinicians rely on laboratory investigations for confirmation of diagnosis and ensuring quality service to patients. The proper management of patients is mainly influenced by accurate $^{1}$ and timely released results from the laboratory ${ }^{2}$. Laboratory errors stand as barriers to achieving this. These errors can occur during the pre- analytical, analytical and post-analytical phases of sample analysis, but pre-analytical errors account for around $70 \%$ of all the errors. ${ }^{2-6}$ The pre-analytical phase includes procedures that start from the clinician requesting a laboratory test on a patient, identification of the patient and sample, patient preparation, collection of the samples, sample handling, transportation, processing and storage ${ }^{7}$. 
Clinical laboratories worldwide have established protocols for sample collection and analysis and several criteria to judge the quality of a sample for analysis. The protocols are essential in guiding appropriate testing and quality results. In the preanalytical phase, a sample is deemed inappropriate for analysis in the following case scenarios: unlabelled specimen, insufficient patient information, haemolysed specimen, wrong container, the inadequate volume of sample, wrong specimen, prolonged transport, clotted sample, no specimen submitted, leaking specimen containers, contaminated request forms, and unpaid specimens ${ }^{1,8}$.

The occurrence of laboratory errors cause delays in sample analysis and prevents the clinicians from providing timely management to their patients which negatively impacts patients' outcome?, especially for the critically ill. A longer stay in the hospital due to delay in sample analysis and treatment also means wasted economic resources on the patient and insurance companies. Sample rejection causes the need to repeat withdrawing more samples and lead to much discomfort, especially for painful procedures like withdrawing blood or cerebrospinal fluid.

Identifying quality indicators is a critical step in the laboratory services that enables the user to quantify, document, monitor, and improve quality ${ }^{2}$. Quality indicators such as patient safety, effectiveness, equity, patient-centeredness, timeliness and efficiency are objective measures to evaluate critical healthcare domains ${ }^{10}$. Determining the suitable quality indicators for the pre-analytical phase is critical as most of the errors occur in this phase of the total testing process (TTP). The initial stages of TTP are neither done in the clinical laboratory nor solely carried out by laboratory personnel. Evaluation, monitoring and improving all the initial process of the TTP is crucial for appropriate patient management ${ }^{11,12}$.

To ensure the quality of results in the laboratory, external quality control is done to measure laboratory performance in pre-analytical, analytical and postanalytical phases. The external quality control is a performance indicator for our quality system and positively contributes to reducing errors in all phases. Sample handling guidelines are available in KCMC clinical laboratory and other hospital departments in order to minimise errors in pre-analytical phases because these errors occur in different places and not only laboratory personnel are involved but also clinicians, nurses and patients ${ }^{13}$. Errors in the preanalytical phase affect both analytical and postanalytical phases ${ }^{14}$. Information on what goes wrong during the pre-analytical phase is vital in deciding where to focus interventions and what improvements can be brought to the currently available guidelines. There is also a laboratory information system (LIS) which is used to record the required details of all samples received in the laboratory, entering test results and authorising their release. Information of
In spite of guidelines on sample handling being available at KCMC clinical laboratory and other hospital departments, there is still an obvious need to improve on the quality of samples provided to the laboratory for analysis. With a better understanding of the sample rejection rate and pre-analytical errors, it will be possible to develop strategies that can help improve the quality of samples submitted to the clinical laboratory department and provide timely management to the patients. This study aimed to assess the overall rate of sample rejection, the reasons and type of sample rejected, the hospital departments they come from, and the clinical laboratory sections they go to.

\section{METHODS}

\section{Study design}

This was a hospital based cross-sectional study. The KCMC Clinical Laboratory Department systematically records every rejected sample using a sample rejection form. The rejection forms are kept in the laboratory's archives and are arranged chronologically.

\section{Setting}

This study was conducted in the Clinical Laboratory department at KCMC Referral Hospital Moshi, Kilimanjaro Region in the Northern zone of Tanzania. This clinical laboratory is accredited as Zonal Reference Laboratory for the Arusha, Dodoma, Kilimanjaro, Singida and Tanga regions. The clinical laboratory is divided into different sections, namely Haematology, Clinical biochemistry, Microbiology, Serology, Blood transfusion, Parasitology and Molecular diagnostics. It receives specimens from outpatient department (OPD) clinics, Casualty, and inpatient departments (Internal Medicine, Obstetrics and Gynaecology, Surgical departments, namely General surgery, Orthopaedics, Urology, ENT, Ophthalmology, and Paediatrics). The laboratory is also a teaching area for students from different programs (Doctor of Medicine, Bachelor of Science in Health Laboratory Sciences, and Diploma in Health Laboratory Sciences).

\section{Data Collection}

Basing on the study's objectives, a data extraction sheet was created and used to manually record the relevant information from the sample rejection forms from the 1st of January to the 31st of December 2016. This will include the patient's hospital number, the hospital department, the time of sample collection and reception, the test requested, the clinical laboratory section, the type of sample and the reason for rejection.

the total number of samples received in the laboratory in 2016 was obtained from LIS. 


\section{Data Analysis}

Data were entered, cleaned and analysed using Statistical Package for Social Science (SPSS); IBM Corporation, New York, USA released in 2012, IBM SPSS Statistics for window, version 20. After extracting information from LIS using data extraction sheets, data were entered in SPSS. Our outcome of interest, number of samples rejected was defined as number of samples rejected during the study period. Rejected samples were coded 1 as samples rejected at the laboratory and 0 as samples that were received and tested in the laboratory. Rejected samples were categorised based on reasons for rejection which included; incomplete patient information (unlabelled samples), samples missing payment details, samples with wrong test, samples with prolonged transport time, haemolysed and clotted samples. Categorical data were summarised by using frequencies and percentages. Graphs and tables were used for data presentation.

\section{Ethical Approval}

Ethical approval was sought from the Kilimanjaro Christian Medical University College (KCMUCo) Ethical Review Committee (Ethical approval reference number: 2398). Permission to access rejected samples data was requested from the Executive Director and Director of Clinical Laboratory Department of KCMC referral hospital. Patients' rejected samples were identified by hospital numbers and no names were mentioned to ensure confidentiality.

\section{RESULTS}

Rate of Sample Rejection and Reasons

A total number of 117181 samples were registered by the KCMC clinical laboratory from the 1st of January to the 31st of December 2016. Out of these, 234 were rejected over this time period for not fulfilling the requirements of the laboratory's collection manual. This means the proportion of sample rejection was $0.2 \%$ and rejection rate was 200 per 100,000 samples. The rates of sample rejection varied in different sections, the highest rates being recorded in the haematology and biochemistry sections. (Table 1 ). Analysis of sample rejection rate in different steps of the pre-analytical phase revealed that most of the sample were rejected at the stage of sample identification and data entry (Table 2 ). In most cases, the reason for sample rejection included unpaid request, clotted and haemolysed samples, and wrong sample collection container (Table 3). Analysis by types of samples (not shown in table) revealed that blood was the most frequently rejected sample 202 $(86.3 \%)$ followed by urine $28(12 \%)$ and stool 4 $(1.7 \%)$.

TABLE 1: Number, Frequency and Rate of Sample Rejection in Different Laboratory Sections

\begin{tabular}{llll}
\hline Laboratory type & Rejected samples & $\begin{array}{l}\text { Frequency of } \\
\text { rejection }\end{array}$ & $\begin{array}{l}\text { Rate of rejection (per } \\
\text { 100,000 samples) }\end{array}$ \\
\hline Haematology & 78 & 33.3 & 67 \\
Biochemistry & 69 & 29.5 & 59 \\
Serology & 34 & 14.5 & 29 \\
Parasitology & 24 & 10.3 & 20 \\
Microbiology & 12 & 5.1 & 10 \\
Blood transfusion & 10 & 4.3 & 9 \\
Molecular biology & 7 & 3.0 & 6 \\
All & 234 & 100.0 & 200 \\
\hline
\end{tabular}

TABLE 2: Rate of Sample Rejection at Each Pre-Analytical Stage ( $N=234$ )

\begin{tabular}{llll}
\hline Pre-analytical stage & $\begin{array}{l}\text { Rejected } \\
\text { samples }\end{array}$ & $\begin{array}{l}\text { Rejection } \\
\text { frequency (\%) }\end{array}$ & $\begin{array}{l}\text { Rejection rate (per } \\
\mathbf{1 0 0 , 0 0 0 ~ s a m p l e s ) ~}\end{array}$ \\
\hline Patient identification & 5 & 2.14 & 4.3 \\
Data entry of the request & 85 & 36.32 & 73 \\
Sample identification & 11 & 4.70 & 94 \\
Sample collection & 53 & 22.65 & 46 \\
Transport of sample & 14 & 5.98 & 12 \\
Suitability of sample & 66 & 28.21 & 56 \\
\hline
\end{tabular}


TABLE 3: Reasons for Sample Rejection

\begin{tabular}{llll}
\hline Description & $\begin{array}{l}\text { Rejected } \\
\text { samples }\end{array}$ & $\begin{array}{l}\text { Rejection } \\
\text { frequency }\end{array}$ & $\begin{array}{l}\text { Rejection rate (per } \\
100,000 \text { samples) }\end{array}$ \\
\hline Clotted sample & 33 & 14.10 & 28 \\
Haemolyzed specimen & 33 & 14.10 & 28 \\
Inadequate sample volume & 9 & 3.85 & 8 \\
Missing patient information & 5 & 2.14 & 4 \\
No specimen submitted & 10 & 4.27 & 9 \\
Prolonged sample transportation & 4 & 1.71 & 3 \\
Unlabeled specimen & 11 & 4.70 & 9 \\
Unpaid sample & 84 & 35.90 & 72 \\
Wrong sample collection container & 36 & 15.38 & 31 \\
Wrong specimen & 8 & 3.42 & 7 \\
Wrong test ordered & 1 & 0.43 & 1 \\
& 234 & 100.00 & 200 \\
\hline
\end{tabular}

\section{FIGURE 1: Frequency of Sample Rejection in Different Laboratory Sections}

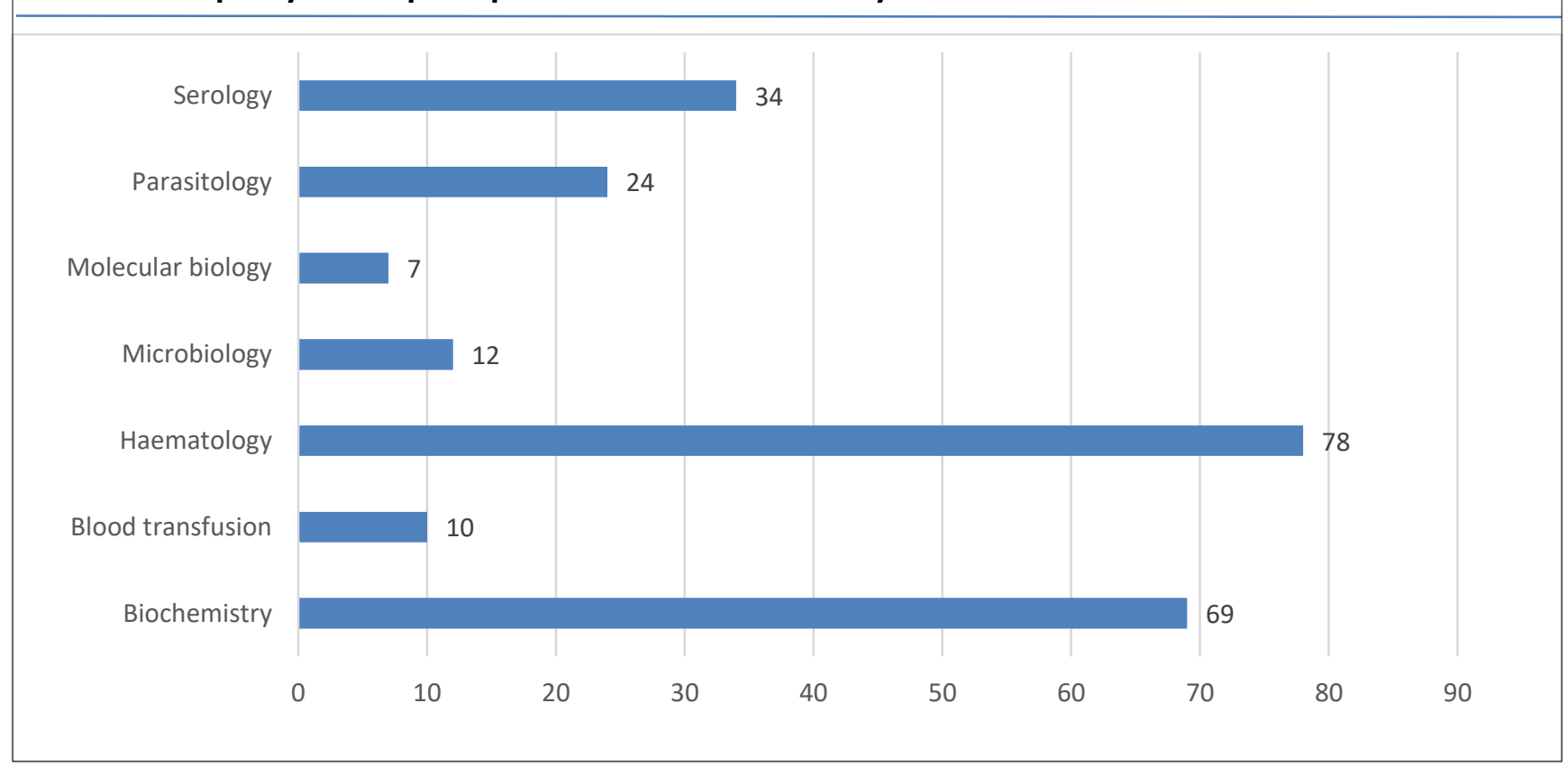

The highest frequency of sample rejection was observed for samples collected in the internal medicine department $47(20 \%)$, with the frequency of rejection from the inpatient medical wards being 32 (13.6\%) which was more than double that of the outpatient clinic $15(6.4 \%)$. The ophthalmology department had the lowest rejection frequency of $5(2.1 \%)$ among all departments. The frequency of sample rejection from different hospital departments are shown in Table 4. Haematology and biochemistry section of clinical laboratory had the highest number of rejected samples (Figure 1). 
TABLE 4: Frequency of Sample Rejection by Hospital Departments ( $N=234)$

\begin{tabular}{lc}
\hline Department & $\mathbf{n}(\%)$ \\
\hline Internal medicine total & $47(20)$ \\
OPD & $15(6.4)$ \\
IPD & $32(13.6)$ \\
Obstetrics and Gynecology total & $33(14.1)$ \\
OPD & $27(11.5)$ \\
IPD & $6(2.6)$ \\
Surgery total & $16(6.8)$ \\
OPD & $9(3.8)$ \\
IPD & $7(3)$ \\
Pediatrics total & $9(3.8)$ \\
OPD & $4(1.7)$ \\
IPD & $5(2.1)$ \\
Dermatology & $15(6.4)$ \\
ENT & $8(3.4)$ \\
Ophthalmology & $5(2.1)$ \\
Urology & $32(13.7)$ \\
Casualty & $40(17.1)$ \\
CCFCC & $6(2.6)$ \\
Diabetic clinic & $6(2.6)$ \\
IDC & $6(2.6)$ \\
SSHS & $11(4.7)$ \\
\hline
\end{tabular}

\section{DISCUSSION}

The proportion of sample rejection was $0.19 \%$ for the year 2016 in KCMC clinical laboratory. This finding is consistent with the findings from China ${ }^{15}$, Q-Probe analysis of 78 clinical laboratories ${ }^{9}$ and close to that conducted in Texas, $\mathrm{USA}^{8}$. The sample rejection frequency in this study is within $(0.1 \%$ to $5.3 \%)$ the reported range from different laboratory settings. $2,6,8,9,15-18$

This study also looked at different causes of sample rejection, and unpaid specimen $84(35.9 \%)$ was the most commonly mentioned reason for sample rejection. This can be possibly explained by a lack of careful inspection of sample collection forms to ensure that the requested sample has been paid for or patients could not afford to pay for laboratory tests. Laboratory personnel at the sample collection area or in phlebotomy should verify a payment stamp on the sample collection forms before collecting a specimen. The limited number of personnel compared to the number of patients that require laboratory services can be another contributing factor to this overlook. An introduction of digital health technology could be the best option to significantly minimise this problem both in the outpatient and inpatient set up. The patients' socioeconomic status is a factor that cannot be overlooked in explaining the lack of payment for received samples. In addition to this, it is important to realise that medical insurance coverage is not available to all patients, limiting their ability to afford laboratory services. A lack of understanding of payment procedures and a readily available tariff of the cost of collection and analysis of different laboratory samples to the patients is another contributing factor to this problem. However, there is a contrast between this study and others where sample clotting was mentioned as the commonest reason for rejection, with a $55.8 \%$ in a Turkish university hospital laboratory ${ }^{2} 43.8 \%$ in Porto Alegre, Brazil ${ }^{5}$ and $51.2 \%$ in Indian ${ }^{1}$. If the laboratory test needs whole blood or plasma, blood sample should be drawn into anticoagulant tubes. Clotting in laboratory blood samples usually occurs when blood is left too long in a tube without an anticoagulant or when it is not adequately mixed in the anticoagulant containing tube $^{19}$. In our study, clotting was ranked second among the reasons for rejection with a frequency of $14.1 \%$, which is also a bit lower than the study done in Ethiopia, which had $16.4 \%$ and higher than the study done in Kenya reported $1.4 \%^{20,21}$. The high frequency of clotted samples might be explained by the lack of in-depth training in phlebotomy, and blood samples transportation for nurses and physicians, as most of clotted samples came from inpatient departments. The high workload for phlebotomists, nurses and physicians is also a contributing factor to these findings.

Blood was the most commonly rejected type of sample with a frequency of $86.3 \%$. This correlates with previous studies conducted in Turkey, China and India. ${ }^{2,15,18}$ The high frequency of rejection of blood samples might be due to different factors which include the difficulty of collecting these samples, the frequency of orders made for these samples by clinicians, and the special requirements for their 
containment and transportation. Among other factors investigated was the origin of the rejected samples. The frequency of sample rejection from different hospital departments was calculated and compared. The internal medicine department had the highest frequency of sample rejection $47(20 \%)$, with most of these coming from its inpatient division. This finding is different from a study conducted in Brazil, which showed that the paediatrics department had a higher sample rejection frequency than other departments 5 . However, concerning inpatient departments, our findings were in line with a study conducted in Turkey that found that adult inpatient departments had a higher frequency of sample rejection than outpatient departments ${ }^{2}$.

The highest sample rejection frequency being from the haematology section $78(33.3 \%)$ and followed by biochemistry $69(29.5 \%)$ can be explained by the fact that these clinical laboratory sections receive a higher load of samples compared to others. It also correlates to a study conducted in Brazil where the haematology section was responsible for most recollection requests $43.6 \%$, followed by the biochemistry section $29 \%{ }^{16}$. Clotted and haemolysed samples are the main reasons for high rejections for haematology and biochemistry sections.

\section{CONCLUSION}

This study allows for a better understanding of the factors that may contribute to laboratory sample rejection in KCMC hospital. Keeping in mind the negative consequences of a delayed sample analysis on a patient's management plan, addressing these factors is invaluable in improving patient care at the preanalytical phase of the laboratory process.

This can be done through an improved quality control system of laboratory processes that will allow for prompt identification of deficiencies in sample management. Therefore, corrective measures can be put in place in a timely manner to allow for improved quality of care.

The high rate of sample rejection due to lack of payment observed in this study can indicate the need for improved government policies that address health expenses coverage.

Identifying vulnerable patients and the availability of support services that help in the payment of their hospital bills can be an initial step in addressing this issue. The introduction of an electronic medical record system that allows easy access to patient information and financial status with regards to hospital expenses can be a possible solution.

Additionally, further training in phlebotomy and blood samples transportation can reduce the high number of samples rejected due to clotting. This can be done through regular, continuous medical education (CME) sessions and joint ward rounds, including clinicians and laboratory personnel. The information gathered from this study can serve as a basis for more research and guide policy makers in implementing these interventions.

\section{REFERENCES}

1. Bhat V, Tiwari M, Chavan P, Kelkar R. Analysis of laboratory sample rejections in the pre-analytical stage at an oncology center. Clin Chim Acta. 2012;413(1516):1203-6.

2. Sinici Lay I, Pınar A, Akbıyık F. Classification of reasons for rejection of biological specimens based on prepreanalytical processes to identify quality indicators at a university hospital clinical laboratory in Turkey. Clin Biochem. 2014:47(12):1002-5.

3. Goswami B, Singh B, Chawla R, Mallika V. Evaluation of errors in a clinical laboratory: a one-year experience. Clin Chem Lab Med. 2010;48(1):63-6.

4. Green SF. The cost of poor blood specimen quality and errors in preanalytical processes. Clin Biochem. 2013;46(13-14):1175-9.

5. Guimarães AC, Wolfart M, Brisolara ML, Dani C. Causes of rejection of blood samples handled in the clinical laboratory of a University Hospital in Porto Alegre. Clin Biochem. 2012;45(1-2):123-6.

6. Jacobsz LA, Zemlin AE, Roos MJ, Erasmus RT. Chemistry and haematology sample rejection and clinical impact in a tertiary laboratory in Cape Town. Clin Chem Lab Med. 2011;49(12):2047-50.

7. Magnette A, Chatelain $M$, Chatelain B, Ten Cate $\mathrm{H}$, Mullier F. Pre-analytical issues in the haemostasis laboratory: guidance for the clinical laboratories. Thromb J. $2016 ; 14: 49$.

8. Cao L, Chen M, Phipps RA, Del Guidice RE, Handy BC, Wagar EA, et al. Causes and impact of specimen rejection in a clinical chemistry laboratory. Clin Chim Acta. 2016;458:154-8.

9. Karcher DS, Lehman CM. Clinical consequences of specimen rejection: a College of American Pathologists QProbes analysis of 78 clinical laboratories. Arch Pathol Lab Med. 2014:138(8):1003-8.

10. Grecu DS, Vlad DC, Dumitrascu V. Quality indicators in the preanalytical phase of testing in a stat laboratory. Laboratory medicine. 2014:45(1):74-81.

11. Plebani M. Quality indicators to detect pre-analytical errors in laboratory testing. Clin Biochem Rev. 2012;33(3):85-8.

12. Plebani M Sciacovelli L Aita A Chiozza ML. Harmonization of pre-analytical quality indicators. Biochem Med (Zagreb). 2014;24(1):105-13.

13. Plebani $M$. The detection and prevention of errors in laboratory medicine. Ann Clin Biochem. 2010;47(2) :10110

14. Majkić-Singh N, Šumarac Z. Quality indicators of the preanalytical phase. J Med Biochem. 2012;31(3):174-83.

15. Lai X, Yang P, Zhang Y, Cao J, Zhang L. Analysis of factors influencing the generation of unqualified clinical samples and measures to prevent this generation. Ann Lab Med. 2012;32(3):216-9

16. Coriolano NL, Silva ICR, Lamounier TA. Analysis of the frequency of biological sample recollections as quality indicators in a clinical laboratory of Distrito Federal, Brazil. JBPML. 2016(ahead):0-

17. Inalegwu A, Phillips S, Datir R, Chime C, Ozumba P, Peters $\mathrm{S}$, et al. Active tracking of rejected dried blood samples in a large program in Nigeria. World J Virol. 2016;5(2):73.

18. Mehrotra A, Srivastava K, Bais P. An evaluation of laboratory specimen rejection rate in a North Indian setting-a cross-sectional study. IOSR JDMS. 2013;7(2):359.

19. Moore G, Knight G, Blann A. Fundamentals of Biomedical Science: Haematology. New York, NY: Oxford University Press; 2010.

20. Kimengech KK, Waithaka SK, Onyuka J, Kigondu CS. Determination of errors that compromise the quality of laboratory service in a tertiary hospital. Asian J Med Sci. $2017 ; 8(1): 64-70$ 
21. Tesfaw HM, Segaye A, Hassen F. Frequency of Specimen Rejection and A ssociated F actors at St. Paul's Hospital Millennium Medical College, Addis Ababa Ethiopia. Multidiscip Healthc. 2015;2(1):1-16.

\section{Peer Reviewed}

Competing Interests: None declared.

Received: 5/2/2020; Accepted: 8/3/2021

Cite this article as: Mosha VV and Kabanyana C. The Rate of Sample Rejection and Pre-Analytical Errors at KCMC Clinical Laboratory in
Moshi, Kilimanjaro. E Afr Sci. 2021;3(1): 118-124. http://doi.org/10.24248/EASci-D-20-00005

(1) Mosha VV et al. This is an open-access article distributed under the terms of the Creative Commons Attribution License, which permits unrestricted use, distribution, and reproduction in any medium, provided the original author and source are properly cited. To view a copy of the license, visit http://creativecommons.org/licens- es/by/4.0/. When linking to this article, please use the following permanent link: http://doi.org/10.24248/EASci-D-20-00005 